\title{
PROTOCOLO DE REVISÃO DE ESCOPO E REVISÃO SISTEMÁTICA NA ÁREA DE
}

\section{ALIMENTOS}

\section{SCOPE REVIEW AND SYSTEMATIC REVIEW PROTOCOL IN THE FOOD AREA}

\author{
Sila Mary Rodrigues Ferreira'; Anabelle Retondario²; Lilian Tanikawa ${ }^{3}$
}

\begin{abstract}
1 - Docente do Departamento de Nutrição e do Programa de Pós-Graduação em Alimentação e Nutrição da Universidade Federal do Paraná (UFPR)

2 - Docente do Departamento de Nutrição da Universidade Federal do Paraná (UFPR)

3 - Discente do Programa de Pós-Graduação em Alimentação e Nutrição da Universidade Federal do Paraná (UFPR)
\end{abstract}

\section{RESUMO:}

O presente material tem como objetivo sugerir um roteiro para guiar o pesquisador no planejamento de Revisão de Escopo e Revisão Sistemática na área de alimentos. A metodologia seguiu o protocolo sugerido pela Cochrane Handbook for Systematic Reviews of Interventions e pelo Joanna Briggs Institute. Esta ferramenta pretende auxiliar na melhoria da qualidade metodológica das revisões na área de alimentos, ao padronizar o método aplicado e buscar maior reflexão sobre o tema de estudo.

Palavras-chave: Revisão sistemática como assunto, Revisão, Alimentos

\section{ABSTRACT:}

This article aims to suggest a script to guide researchers in planning Scope Reviews and Systematic Reviews in the food science field. We follow the Cochrane Handbook for Systematic Reviews of Interventions methodology, from Joanna Briggs Institute. This tool intends to help researchers to improve methodological quality in their reviews in the food science field when standardizing the method applied and search bring reflections about the study topic.

Keywords: Sistematic Review as a topic, Review, Food

\section{INTRODUÇÃO}

O conhecimento científico se inicia por meio de buscas sobre o tema que se deseja conhecer. É mediante dessas buscas, também, que são identificados o que se sabe, as controvérsias e o que ainda precisa ser esclarecido, consequentemente, possibilidades de pesquisas. Assim, a revisão de literatura é o procedimento de organizar as buscas e os resultados, para vislumbrar um melhor panorama e uma discussão mais embasada dos achados (INGRAM et al., 2006; BOTELHO; CUNHA; MACEDO, 2011).

Segundo Greenhalgh (1997), revisão sistemática é a revisão realizada com metodologia clara e reprodutível para obter a síntese de estudos com dados primários que tenham seus objetivos, materiais e métodos claramente expostos. Com ela, busca-se 
compilar os resultados dos estudos encontrados para propor uma evidência científica de determinada qualidade (ARKSEY; O’MALLEY, 2005)

Já a revisão de escopo tem o objetivo de, com igual metodologia rigorosa e reprodutível, mapear o estado da arte sobre determinado tema, descrevendo os achados. Não se objetiva compilar os achados ou avaliar criticamente a qualidade da evidência encontrada (ARKSEY; O'MALLEY, 2005).

Diante do exposto, a construção desse material teve como objetivo a sugestão de padronização de protocolo de Revisão de Escopo e Revisão Sistemática na área de alimentos, uma vez que as diretrizes e os acrônimos sugeridos pela Cochrane Handbook for Systematic Reviews of Interventions e pelo Joanna Briggs Institute (PETERS et al., 2017) são dirigidos para pesquisas com desfechos em evidências em humanos. No entanto, entende-se que a metodologia empregada nessas plataformas pode ser aplicável para quaisquer áreas do conhecimento, agregando rigor metodológico às pesquisas. A diferença da metodologia dessas plataformas reside, além da publicação do artigo final (GAFFEY et al., 2017), no fato de que os autores devem registrar inicialmente o nome da pesquisa na plataforma e publicar o protocolo previamente (GAFFEY et al., 2017; ELLWOOD et al., 2018). Tais procedimentos pretendem melhorar a qualidade metodológica das revisões, ao buscar promover maior reflexão sobre o tema de estudo e padronizar o método aplicado.

Assim, o presente material apresentará um roteiro com o intuito de guiar o pesquisador no planejamento de revisão sistemática ou de escopo, para obtenção de um protocolo padronizado.

\section{METODOLOGIA}

Trata-se de uma sistematização de proposta para protocolos de revisão de escopo e revisão sistemática na área de alimentos. A construção do presente roteiro proposto está baseada no esquema da FIGURA 1 e no método Systematic Search Flow (FERENHOF; FERNANDES, 2016), sugerido por Jesson, Matheson e Lacey, (2011), conforme mostra a FIGURA 2. 


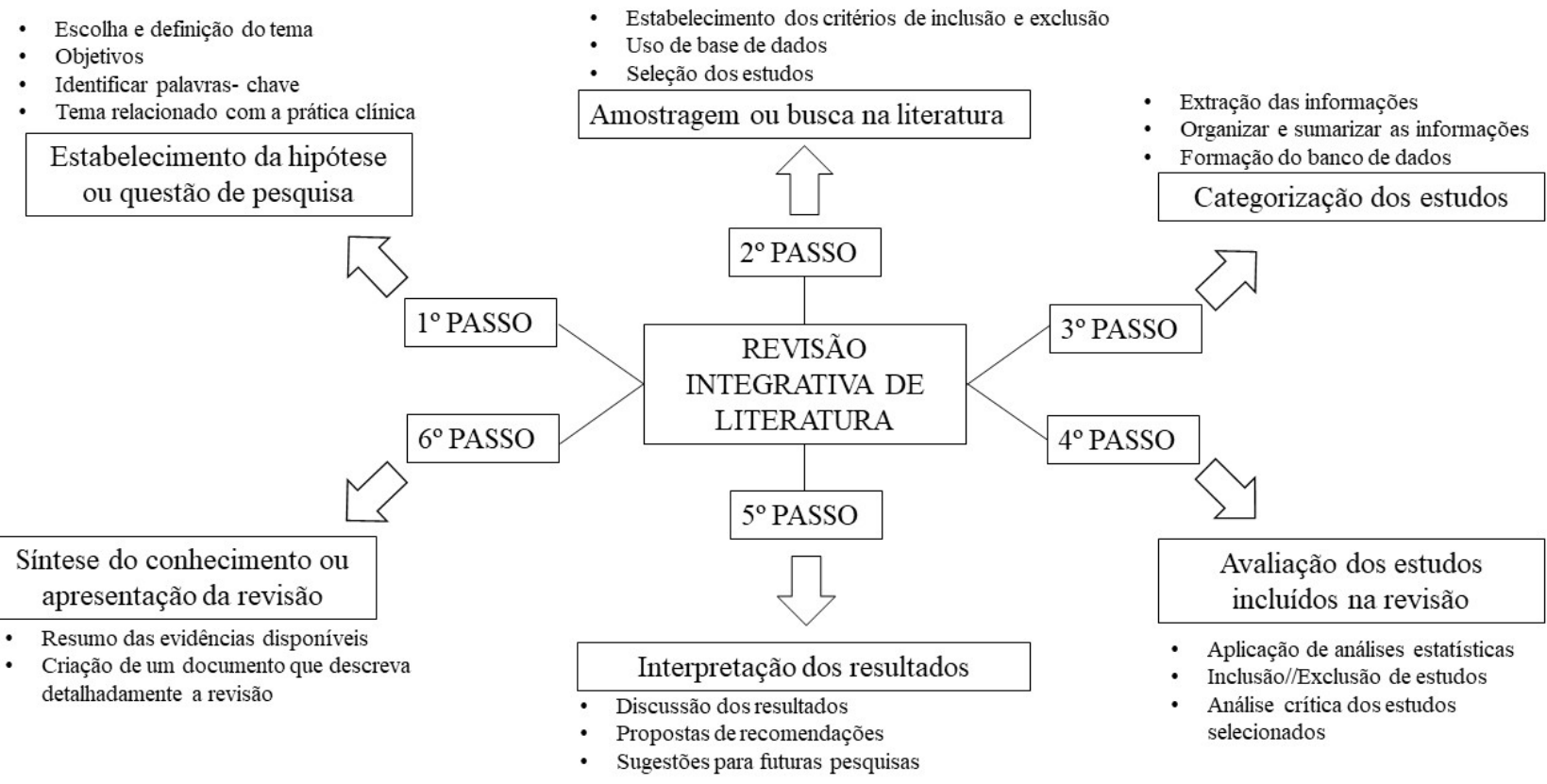

FIGURA 1 - PASSOS DA REVISÃO DE LITERATURA.

Fonte: (MENDES; SILVEIRA; GALVÃO, 2008).

Conforme observado na FIGURA 1, a revisão deve iniciar com uma hipótese e/ou pergunta de partida bem definidas, a fim de delimitar o tema pesquisa. A definição deve ser embasada em revisão prévia da literatura, pois somente assim o pesquisador terá conhecimento do campo de pesquisa e, consequentemente, da lacuna que ainda precisa ser preenchida. Em seguida, devem ser definidos critérios de inclusão e exclusão, as bases de dados pesquisadas e realizada a busca na literatura. Num terceiro passo, os estudos selecionados devem ser organizados para extração de dados sistemática, previamente padronizada com base nas perguntas de partida, para formação do banco de dados da pesquisa. $\mathrm{O}$ quarto passo se refere à avaliação dos estudos incluídos na revisão, o que permitirá refletir sobre a qualidade dos achados da revisão. O quinto passo se debruça sobre os dados compilados para sua interpretação à luz do conhecimento científico e discussão dos resultados. Com isso é possível tirar conclusões (ainda que preliminares, em certos casos) e sugerir novas investigações a fim de cobrir lacunas evidenciadas. Por fim, o sexto passo trata da organização de todas as informações da revisão (desde como foi definida e conduzida até os resultados e conclusões) para divulgação no meio científico, usualmente em formato de artigo em periódicos indexados. 


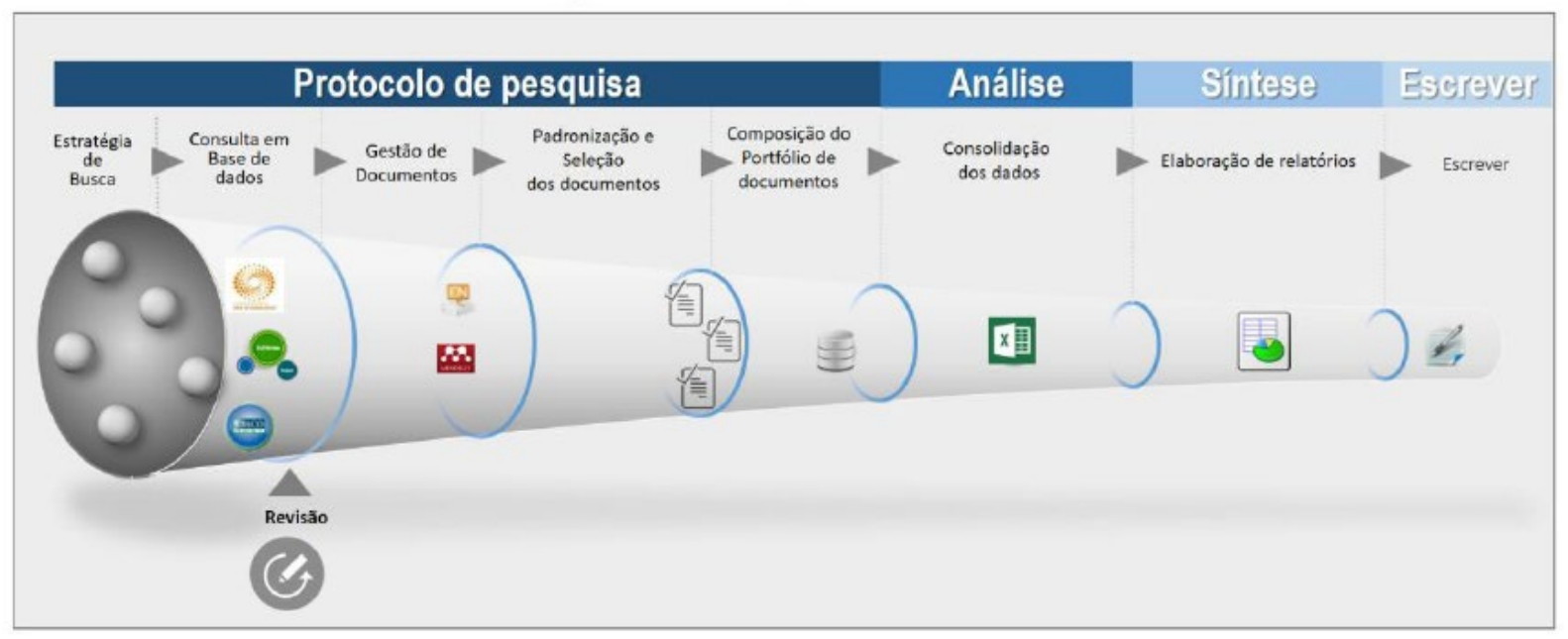

FIGURA 2 - MÉTODO SYSTEMATIC SEARCH FLOW

Fonte: (FERENHOF; FERNANDES, 2016).

A Figura 2 apresenta o fluxo da busca sistemática para revisões. O método se divide em 4 fases: protocolo de pesquisa, análise, síntese e escrever. A primeira fase é dividida em 5 atividades relacionadas ao protocolo de pesquisa; a) definição da estratégia de busca, b) consulta (busca) nas bases de dados, c) gestão das referências mediante a transferência dos dados a um programa de gerenciamento, d) análise dos dados de maneira padronizada, baseada nos critérios de inclusão e exclusão, para seleção dos artigos a serem incluídos na revisão e, por último, e) composição de um portfólio com os artigos selecionados.

\subsection{ROTEIRO DE PROTOCOLO DE REVISÃO SISTEMÁTICA OU DE ESCOPO}

\subsubsection{Identificação: título da pesquisa e autores}

O protocolo deve iniciar com a identificação, onde constem informações como título da revisão, autores envolvidos de acordo com as normas da revista pré-selecionada para divulgação dos resultados. O título da pesquisa deve ser claro e objetivo, dando ao leitor informações concisas e suficientes sobre o tema da pesquisa e o tipo de estudo. Por exemplo: "Ácido Oxálico em Plantas Alimentícias não-convencionais: Uma revisão de escopo".

A lista de autores deve apontar quem serão os pesquisadores envolvidos na pesquisa. Também já se pode aproveitar a elaboração do protocolo para definir os papéis de cada pesquisador, como por exemplo: Pesquisador A: responsável pelo planejamento da pesquisa, redação do protocolo, busca sistemática, seleção dos estudos, 
compilação e interpretação dos resultados, redação do trabalho final (TCC, dissertação etc.), redação do manuscrito, submissão do manuscrito em periódico indexado (esses são exemplos de etapas usualmente executadas pelo aluno que está desenvolvendo seu projeto); Pesquisador B: participação no planejamento da pesquisa, redação do protocolo, busca sistemática, seleção dos estudos, contribuição na interpretação dos resultados e redação do manuscrito final (esses são exemplos de etapas usualmente executadas um aluno que fará dupla com o aluno-pesquisador $\mathrm{A}$ ); Pesquisador $\mathrm{C}$ : orientação em todas as fases da pesquisa (planejamento, protocolo, busca sistemática, seleção dos estudos, compilação e interpretação dos resultados), terceiro revisor quando os Pesquisadores $\mathrm{A} \mathrm{e}$ $B$ não chegaram a um consenso, revisão crítica do trabalho final e do manuscrito para submissão (esses são exemplos de etapas usualmente executadas pelo orientador do aluno-Pesquisador $\mathrm{A}$ ).

Outros possíveis pesquisadores, do grupo de pesquisa, podem colaborar com a revisão. Alguns exemplos de etapas que podem ser executadas pelos demais autores são: coorientação em todas ou em determinadas fases da pesquisa, análise e interpretação dos resultados, elaboração de gráficos e tabelas, terceiro revisor quando os autores Pesquisadores A e B não chegaram a um consenso, revisão crítica do trabalho final e do manuscrito para submissão.

A revista para publicação deve ser selecionada com base em leituras prévias, quando se poderá identificar quais periódicos publicam o tema da pesquisa ou temas similares e, especialmente, se aceitam submissão de revisões (sejam sistemáticas, de escopo, integrativas...) que não sejam na modalidade "a convite". Importante observar, também, se o periódico possui taxas para submissão e/ou publicação dos estudos. No protocolo, é importante listar cerca de três periódicos, juntamente com sua classificação no Qualis Periódicos da Capes, em ordem de preferência (do maior para o menor Qualis).

\subsubsection{Resumo}

O resumo deve apresentar de forma sintetizada todo protocolo proposto. Recomenda-se que seja a última etapa a ser realizada, para que os autores tenham a dimensão de todo trabalho. É comum que a quantidade de palavras seja limitada a 250 com espaços. No protocolo, não há necessidade de contar as palavras, mas tenha certeza de que o texto está direto, claro e conciso. Reduzir o número de palavras para o limite pode ser um exercício para o momento da redação do manuscrito para submissão. 


\subsubsection{Palavras-chave}

Em relação às palavras-chave, devem ser listadas de 3 a 6 palavras que descrevam a pesquisa, o tema principal e podem identificar o tipo de estudo. "Revisão" e "Revisão sistemática" são exemplos de termos indexados que identificam esse tipo de pesquisa. As palavras-chave devem ser identificadas com bases dos termos de busca, como por exemplo as bases da Food Science \& Technology Abstracts (FSTA, http://www.ifis.org/fsta), da National Center for Biotechnology Information (NCBI, https://www.ncbi.nlm.nih.gov/mesh/) e da Biblioteca Virtual em Saúde (BVS, https://decs.bvsalud.org/).

\subsubsection{Introdução}

A introdução do protocolo deve abranger o estado da arte, apresentando o que já se sabe, o controverso e o que ainda não está esclarecido sobre o tema, de modo a deixar claro ao leitor a lacuna do conhecimento. Ela deve ser objetiva e sucinta. Não deve ser tão extensa e detalhada quanto a revisão de literatura de um TCC ou uma dissertação, mas deve apresentar o mais importante, de maneira lógica e concisa.

É comum que alguns periódicos "sugiram" o máximo de cinco parágrafos para a introdução. No protocolo, não é necessário se prender ao número exato de parágrafos, mas reduzir e manter dentro ou próximo do recomendado pelas revistas pode ser um bom treino. Também não se devem ser feitos parágrafos muito longos e cansativos. Deve-se procurar ser objetivo, direto e claro na exposição das ideias.

\subsubsection{Pergunta norteadora ou objetivo}

A fim de realizar uma pesquisa relevante, que gere desdobramentos positivos na prática profissional, é necessário que o pesquisador consiga elaborar questões importantes dentro da temática escolhida e que sejam factíveis em sua realidade profissional.

A pesquisa científica é um processo sistemático que tem por finalidade gerar novos conhecimentos e/ou refutar ou corroborar conhecimento preexistente. Por isso, a pergunta norteadora ou pergunta científica deve ser a etapa entendida como objeto de estudo ou objeto da pesquisa. Assim, a pergunta norteadora é importante porque, como o próprio nome diz, ela norteia todo o trabalho, ou seja, ela evita que o pesquisador desvie do tema, além de diminuir o risco de viés do estudo. Portanto, a pergunta pode ser colocada na forma interrogativa e deve ser específica, clara, explícita e operacional(GRAZIOSI, MARIA 
ELISABETE SALVADOR; LIEBANO, RICHARD ELOIN; NAHAS, 2010). O texto deve ser claro e enxuto a fim de explicar o objeto proposto, conforme mostra o texto a seguir: "O protocolo de revisão sistemática foi realizado para verificar a quantidade de oxalato presente nas partes morfológicas de Plantas Alimentícias Não Convencionais - PANC”.

\section{Exemplos de pergunta norteadora:}

- Quais PANC têm ácido oxálico na sua composição?

- Quais partes das PANC têm maior quantidade de ácido oxálico?

\subsubsection{Método}

O método deve ser descrito de maneira clara e objetiva, embasado na literatura científica e devidamente referenciado.

\subsubsection{Critérios de Inclusão ou Critérios de Elegibilidade}

As perguntas de pesquisa devem ser utilizadas para nortear as buscas e definir os critérios de inclusão. Tais critérios devem procurar abranger a maior quantidade possível de estudos que versem sobre os temas principais e precise esclarecer ou contextualizar conhecimentos relacionados à pergunta norteadora.

Considerando as perguntas propostas no exemplo (Quais PANC têm ácido oxálico na sua composição? Quais partes das PANC têm maior quantidade de ácido oxálico?), os critérios de elegibilidade devem procurar abranger todos os estudos que versem sobre PANC, partes de PANC e ácido oxálico.

\subsubsection{Critérios de Exclusão}

Nesse item é importante deixar claro quais estudos, dentre os previstos nos critérios de inclusão, serão excluídos. Importante observar que somente os estudos incluídos num primeiro momento podem ser posteriormente excluídos. Seguindo o mesmo exemplo, no qual seriam incluídos estudos que versem sobre PANC, partes de PANC e ácido oxálico, possíveis critérios de exclusão seriam: estudos realizados com frutas, estudos em idiomas que não português, inglês e espanhol, e estudos que apresentarem os resultados somente em gráficos e/ou figuras e, mesmo após contato com os autores, não seja possível extrair os dados necessários para a revisão. 


\subsubsection{Desenho do Estudo}

Todos os procedimentos comuns às revisões sistemáticas (testes de busca durante a elaboração do protocolo, coleta de dados, extração de dados, síntese, análise e discussão dos resultados, e conclusões) devem seguiras recomendações preconizadas pela Cochrane (Cochrane Handbook for Systematic Reviews of Interventions) e pela diretriz Joanna Briggs Institute (JBI, 2015; PETERS et al, 2017), atendendo à padronização necessária para a elaboração de uma revisão sistemática.

\subsubsection{Estratégia de Busca}

A estratégia de busca tem como objetivo encontrar estudos publicados e não publicados, sem restrição de data e idioma a respeito do tema em estudo. Para nortear as buscas, são sugeridos construtos que têm como referência à(s) pergunta(s)norteadora(s). A partir dos construtos são indicados as "strings", palavras-chave, sinônimos, nome científico, popular, conforme Moraes et al. (2019) e Badger et al. (2000). Exemplos de perguntas e constructos podem ser observados no QUADRO 1 e na FIGURA 3.

Quadro 1 - Exemplo de tabela para esquematização dos parâmetros de pesquisa:

\begin{tabular}{|l|l|l|l|}
\hline $\begin{array}{c}\text { Perguntas de } \\
\text { pesquisa }\end{array}$ & Constructos & $\begin{array}{l}\text { Campos da } \\
\text { pesquisa } \\
\text { identificados } \\
\text { nos constructos }\end{array}$ & \multicolumn{1}{|c|}{ Estratégia de busca (strings) } \\
\hline $\begin{array}{l}\text { Quais PANC têm } \\
\text { ácido oxálico na } \\
\text { sua composição? }\end{array}$ & $\begin{array}{l}\text { Presença de } \\
\text { ácido oxálico } \\
\text { em quaisquer } \\
\text { PANC }\end{array}$ & $\begin{array}{l}\text { Ácido oxálico } \\
\text { PANC }\end{array}$ & $\begin{array}{l}\text { (Ácido oxálico OR oxalic acid OR } \\
\text { Oxalates) AND (edible wild plants OR } \\
\text { plantas alimentícias não convencionais } \\
\text { OR plantas comestíveis OR plants, } \\
\text { edible OR underutilized plants }\end{array}$ \\
\hline $\begin{array}{l}\text { Quais partes das } \\
\begin{array}{l}\text { PANC têm maior } \\
\text { quantidade de ácido } \\
\text { oxálico? }\end{array}\end{array}$ & $\begin{array}{l}\text { Ácido oxálico } \\
\text { nas diferentes } \\
\text { partes das PANC }\end{array}$ & $\begin{array}{l}\text { Ácido oxálico } \\
\text { Diferentes partes } \\
\text { de PANC } \\
\text { PANC }\end{array}$ & $\begin{array}{l}\text { Axcido Oxálico OR Oxalic acid OR } \\
\text { oxalates) AND (Edible wild plants OR } \\
\text { plantas alimentícias não convencionais } \\
\text { OR plantas comestíveis OR plants, } \\
\text { edible OR underutilized plants) AND } \\
\text { (caules de planta OR plant stems OR } \\
\text { folhas de planta OR plant leaves OR } \\
\text { rizoma OR rhizome OR raízes de } \\
\text { plantas OR plant roots OR tubérculos } \\
\text { OR plant tubers OR sementes OR } \\
\text { seeds OR nozes OR nuts OR flores } \\
\text { OR flowers) }\end{array}$ \\
\hline
\end{tabular}

Fonte: Elaborado pelas autoras com base em (DE MORAES et al., 2019). 
Deve-se inserir, no protocolo de revisão, uma tabela com os parâmetros da pesquisa, onde se demonstrará que as perguntas de pesquisa darão origem aos construtos que, por sua vez, vão possibilitar a geração das strings.

No QUADRO 1, observa-se que a primeira pergunta de pesquisa pretende identificar as PANC que contêm um determinado componente (ácido oxálico) em sua composição. Para tal questionamento, conclui-se que o construto será a presença de ácido oxálico em PANC. Para estruturar a string de busca, deve-se atentar para todos os campos de pesquisa identificados no construto, neste caso: o ácido oxálico e as PANC. Quais as palavras-chave para localizar estudos que trataram sobre "ácido oxálico"? Essas palavras devem ser buscadas em bases de palavras-chave, como Food Science \& Technology Abstracts (FSTA, http://www.ifis.org/fsta), da National Center for Biotechnology Information (NCBI, https://www.ncbi.nlm.nih.gov/mesh/) e da Biblioteca Virtual em Saúde (BVS, https://decs.bvsalud.org/).

Além das palavras-chave catalogadas, quais outros possíveis termos sinônimos poderiam identificar tais estudos? Estes outros são termos comumente utilizados em estudos publicados sobre o assunto, que podem ser identificados pela leitura e apropriação do tema. Por fim, para estruturação da string, devem ser utilizados os operadores booleanos OR e AND. Todas as palavras-chave de um determinado campo devem ser integradas pelo operador OR, criando, assim, blocos de termos de busca. Os blocos de termos, então, devem ser ligados pelo operador

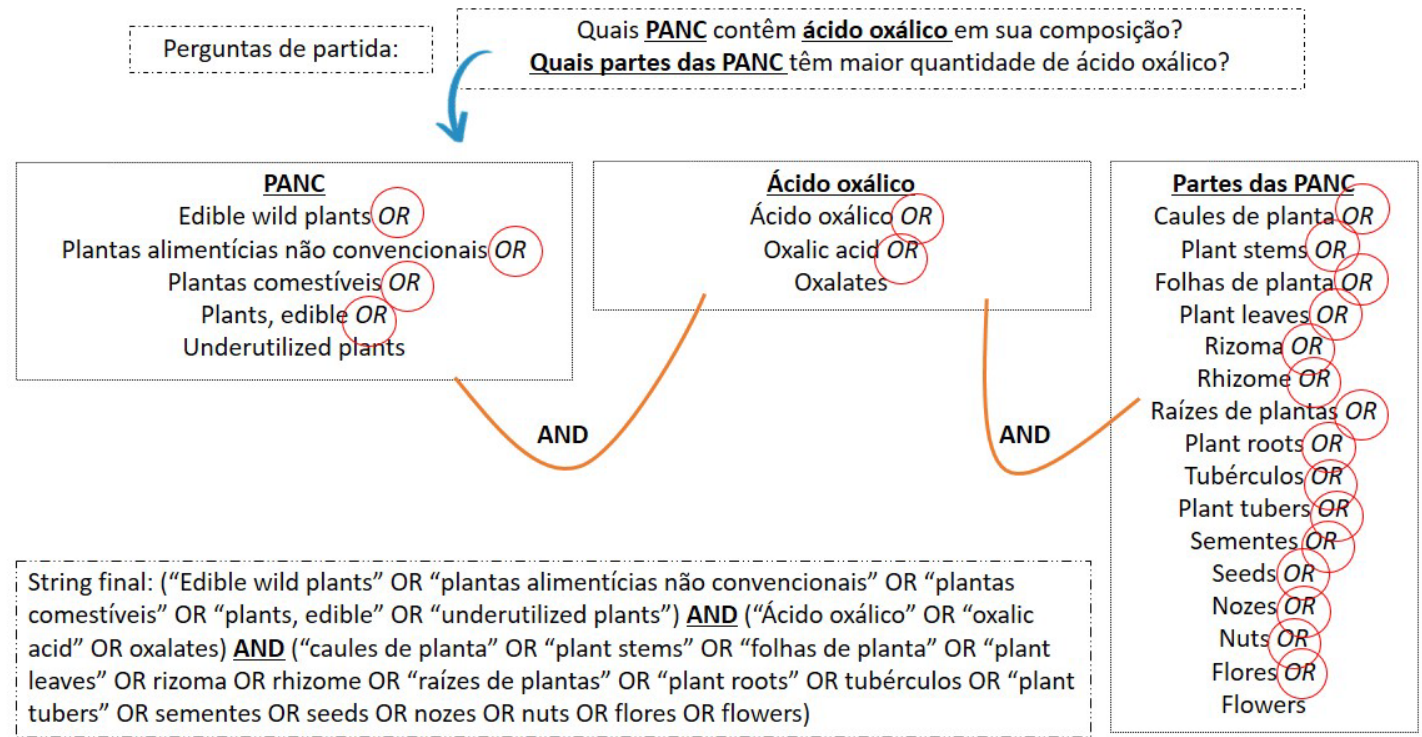

FIGURA 3 - EXEMPLO DE CONSTRUÇÃO DA STRING DE BUSCA

Fonte: Elaborado pelas autoras com base em (MORAES et al., 2019; BADGER et al., 2000). 
Dois pontos são dignos de nota: a) a extensão da estratégia de busca, composta por vários descritores/palavras-chave de três blocos campos e b) que diferentes bases de dados exigem diferentes estratégias de busca, em virtude das suas particularidades de busca avançada. Por isso, é importante ter o auxílio de um profissional de biblioteconomia, que entenda e acompanhe as escolhas das palavras-chave e auxilie na composição das diferentes strings para cada base de dados.

\subsubsection{Fontes de Informações}

As bases de dados onde serão realizadas as buscas devem ser pré-definidas no protocolo da revisão. Elas devem incluir o maior número possível de bases relevantes para o tema investigado. São exemplos: Biblioteca Virtual em Saúde (BVS), PubMed, Embase, Portal de periódicos CAPES, FSTA - Food Science and Technology Abstracts (EBSCO), Science Direct, Scopus, Web of Science, SPORTDiscus. Além dessas, deve ser realizada uma busca por estudos não publicados e possivelmente elegíveis, na literatura cinzenta, como Open Grey, DART-Europe E-thesis Portal e no Google Scholar. Deve estar previsto no protocolo da revisão sistemática que as buscas serão realizadas em duplicata, por pesquisadores independentes, a fim de confrontar o número de estudos encontrados em cada base de dados.

\subsubsection{Seleção dos Estudos}

A seleção de estudos a serem incluídos na revisão sistemática deve seguir as recomendações preconizadas pela Cochrane (Cochrane Handbook for Systematic Reviews of Interventions) e pela diretriz Joanna Briggs Institute (JBI, 2015, PETERS et al, 2017). Após a busca nas bases de dados, por dois revisores independentes, os artigos encontrados poderão ser agrupados e transportados para um software gerenciador de referências, como Zotero ${ }^{\circledR}$, Endnote ${ }^{\circledR}$ ou Mendeley ${ }^{\circledR}$, onde são facilmente descartadas as duplicatas. A FIGURA 4 apresenta etapas da seleção dos estudos. 


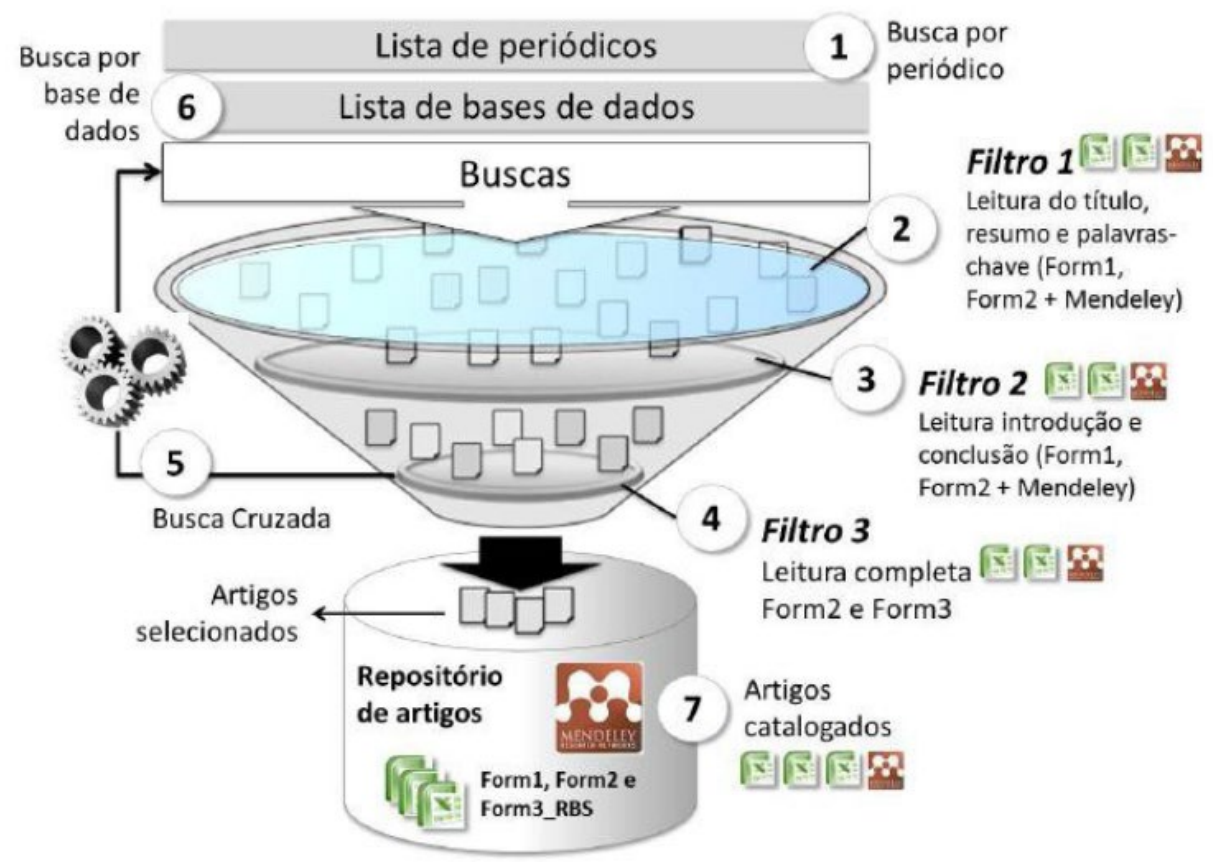

FIGURA 4 - ETAPAS DE SELEÇÃO DOS ARTIGOS A SEREM INCLUÍDOS EM UMA REVISÃO SISTEMÁTICA.

Fonte: (CONFORTO; AMARAL; SILVA, 2011).

Com a lista de referências sem duplicatas, a seleção dos estudos por parte dos revisores deve iniciar com a leitura dos títulos e resumos (Filtro 1), a fim de pré-selecionar, com base nos critérios de inclusão e exclusão previamente definidos, artigos potencialmente elegíveis. Essa etapa pode ser realizada no próprio software de referências ou exportando os resultados para softwares de edição de texto (.rtf) e/ou planilhas (.xml) usando o output style Annotated, modo no qual serão exportadas informações básicas do artigo (autor, ano, título, publicação) e o resumo.

Os estudos pré-selecionados devem, então, ser recuperados na íntegra para leitura completa. Para melhor controle das etapas de seleção, recomenda-se que seja criado um banco em software de edição de planilhas com a lista de artigos para leitura, no qual deve ser registrada qualquer informação pertinente, como motivo de exclusão do artigo préselecionado e/ou qualquer dúvida quanto à sua inclusão na revisão. Em seguida, deve ser realizada a leitura da introdução e conclusão desses artigos (Filtro 2), para verificar sua pertinência ao tema e, posteriormente deve ser realizada a leitura completa dos artigos (Filtro 3) para definir por sua inclusão ou não na revisão.

Pode-se observar que, desde a lista inicial até este momento da pesquisa, a busca passa por um funil (FIGURA 4), reduzindo substancialmente o número de estudos entre os 
localizados na busca e os definidos como elegíveis. Nesse ponto, as listas de referências de todos os artigos selecionados devem ser avaliadas criteriosamente em busca de possíveis artigos elegíveis que não foram identificados nas buscas às bases de dados. Se algum artigo for identificado, ele deve passar pelos 3 Filtros a fim de verificar sua elegibilidade.

Quaisquer divergências que surgirem entre os revisores, em qualquer uma das etapas do processo de seleção, deverão ser resolvidas por meio de discussão entre ambos ou com auxílio de um terceiro revisor.

Os resultados devem ser apresentados em fluxograma, conforme sugerido pelo Preferred Reporting Items for Systematic Reviews and Meta-Analyses (PRISMA) (MOHER, DAVID; LIBERATI, ALESSANDRO; TETZLAFF, JENNIFER; ALTMAN, 2009). Um exemplo de fluxograma está apresentado na FIGURA 5.

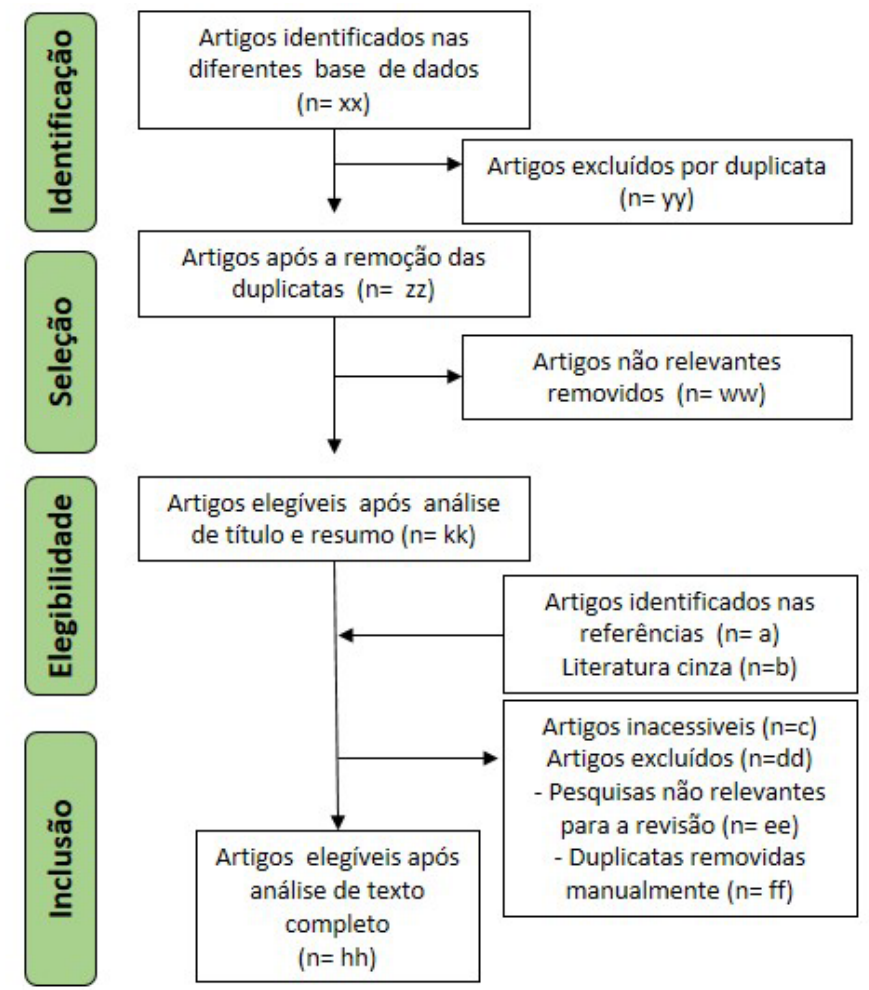

FIGURA 5 - EXEMPLO DE DIAGRAMA DE FLUXO

Fonte: Elaborado pelas autoras com base em MOHER et al., 2009.

\subsubsection{Avaliação da Qualidade Metodológica}

Os estudos elegíveis passam por processo de avaliação crítica da qualidade 
metodológica que pode ser denominada análise bibliométrica. Essa avaliação é realizada por dois revisores de maneira independente. Quaisquer divergências que surjam entre os revisores são resolvidas por meio de diálogo ou mediante um terceiro revisor. Os resultados da avaliação crítica são relatados em forma narrativa e em tabelas. Sempre que possível, os estudos, independentemente da qualidade metodológica dos resultados, devem ser submetidos à extração e síntese de dados.

O portfólio dos artigos analisados compreende o padrão da escrita e possibilidade de análise estatística (PILKINGTON; MEREDITH, 2009). A análise deve contemplar: a) A técnica de investigação, que tem a finalidade de analisar o tamanho, crescimento e distribuição bibliográfica do estudo no campo do conhecimento; b) análise bibliométrica que consiste na análise dos indicadores do artigo, como: base de dados onde o encontrou; número de citações (encontrar na Base de Dados usada), impacto do artigo (http://www.plos.org/plos-one-measuringarticleimpact/), peso do periódico (http://www.journalmetrics.com/ e http://scimagojr.com/) (MEDEIROS et al., 2015); c) Análise dos gráficos, figuras e tabelas a fim de retirada dos dados quantitativos. Em suma, a análise ajuda a estabelecer relações e significância entre os termos da busca, identificando de forma sistemática o conjunto de fatores relevantes à pesquisa (FERENHOF; FERNANDES, 2016).

\subsubsection{Extração e Síntese dos Dados}

A avaliação dos estudos incluídos na revisão e aplicação da análise estatística deve seguir os critérios de inclusão e exclusão; análise crítica dos estudos selecionados. Durante a revisão, os dados dos artigos incluídos são registrados em planilhas no Microsoft Office Excel®. Além das informações básicas (dados dos autores, da publicação, abstract), deve haver um destaque do principal achado do artigo. Caso haja alguma discordância entre os dados extraídos pelos dois revisores, deve ser resolvida por diálogo entre ambos ou, caso necessário, com um terceiro revisor. Na falta ou necessidade de algum dado adicional, os autores dos artigos originais poderão ser contatados por e-mail. Exemplo de planilha para extração e síntese dos dados está apresentado na FIGURA 6.

\begin{tabular}{|c|c|c|c|c|c|c|c|c|c|c|}
\hline \multicolumn{11}{|c|}{ Data da extração: } \\
\hline Autores & Ano & Revista & $\begin{array}{l}\text { Contato autor } \\
\text { correspondente }\end{array}$ & $\begin{array}{c}\text { Objetivo } \\
\text { geral }\end{array}$ & $\begin{array}{l}\text { Tipo de } \\
\text { estudo }\end{array}$ & $\begin{array}{l}\text { Objeto da pesquisa } \\
\text { (nome científico) }\end{array}$ & Desfecho & $\begin{array}{c}\text { Testes } \\
\text { estatísticos }\end{array}$ & $\begin{array}{l}\text { Principais } \\
\text { resultados }\end{array}$ & $\begin{array}{l}\text { Conclusão } \\
\text { do artigo }\end{array}$ \\
\hline & & & & & & & & & & \\
\hline & & & & & & & & & & \\
\hline
\end{tabular}

FIGURA 6 - EXEMPLO DE TABELA DE EXTRAÇÃO DE DADOS Fonte: Elaborado pelas autoras. 


\subsubsection{Análise e Interpretação dos Resultados}

A análise dos resultados deve ser realizada de forma crítica, procurando na literatura explicações para os resultados conflitantes nos diferentes estudos. Dentre as abordagens, o revisor pode aplicar análise estatística e meta-análise (FALLAH; SARMAST; JAFARI, 2020; TOFANELLI; WORTMAN, 2020). A competência do revisor em relação ao tema (como conhecimento prévio) contribui para a avaliação crítica dos estudos, auxilia na tomada de decisão para a utilização dos resultados da pesquisa e para gerar conclusões e recomendações práticas (MENDES; SILVEIRA; GALVÃO, 2008).

A interpretação consiste na síntese do conhecimento, discussão dos resultados, propostas e recomendações e sugestões para futuras pesquisas. Cada achado deve ser analisado à luz da literatura e discutido com base na ciência. "O que isso significa? E o que outros autores já descobriram de relevante sobre isso?" O que esse achado representa para o avanço do conhecimento, para a resposta da pergunta norteadora ou para criar uma nova lacuna para futuras pesquisas.

\subsubsection{Cronograma}

O cronograma da revisão deve ser pautado na plausibilidade. O pesquisador responsável deve refletir sobre o prazo para conclusão do trabalho (bancas, submissão de manuscritos etc.) e o tempo disponível dos revisores para seleção e extração dos artigos, para posterior análise e redação. Importante observar que o tempo máximo entre a realização da busca e a submissão do manuscrito deve ser de 180 dias. Após esse período, recomenda-se a atualização da busca para que o manuscrito não seja submetido com dados defasados. Para facilitar o planejamento das atividades e gestão de projetos deve ser utilizado um cronograma. Assim, como ferramenta pode ser utilizado o Diagrama de Gantt (CLARK,1922, ROSALEM, 2021),no qual são definidas as atividades (no eixo vertical) e na linha temporal (eixo horizontal) quando serão realizadas (SLACK; BRANDON-JONES; JOHNSTON, 2018).

\subsubsection{Pontos fortes e limitações}

$\mathrm{Na}$ seção de pontos fortes e limitações, devem estar apresentadas reflexões, considerando que toda pesquisa deve ser relevante, caso contrário, por que fazê-la? Então, 
é importante refletir sobre os pontos fortes da revisão, por que será importante e o que deverá trazer de novo para o arcabouço teórico sobre o tema, o avanço do conhecimento na área. Da mesma maneira, toda pesquisa é imperfeita. Há sempre um método ou um desenho que poderia ser aprimorado, mas estava fora do alcance dos pesquisadores no momento da pesquisa.

\section{CONSIDERAÇÕES FINAIS}

Esse material foi realizado com objetivo de orientar pesquisadores na elaboração de protocolo de Revisão de Escopo e Revisão Sistemática na área de alimentos tendo como metodologia o protocolo sugerido pela Cochrane Handbook for Systematic Reviews of Interventions e pelo Joanna Briggs Institute. Espera-se que o roteiro contribua para sistematização e otimizar da busca e resultar em artigo de melhor qualidade.

\section{REFERÊNCIAS}

ARKSEY, H.; O'MALLEY, L. Scoping studies: Towards a methodological framework. International Journal of Social Research Methodology: Theory and Practice, v. 8, n. 1, p. 19-32, 2005.

BADGER, D. et al. Should all literature reviews be systematic? Evaluation and Research in Education, v. 14, n. 3-4, p. 220-230, 2000.

BOTELHO, L. L. R.; CUNHA, C. C. de A.; MACEDO, M. O Método Da Revisão Integrativa Nos Estudos Organizacionais. Gestão e Sociedade, v. 5, n. 11, p. 121, 2011.

CONFORTO, E. C., AMARAL, D. C.; SILVA, S. L. da. Roteiro para revisão bibliográfica sistemática: aplicação no desenvolvimento de produtos e gerenciamento de projetos. Resumos. 2011.

CLARK, W. The Gantt Chart: A Working Tool of Management. New York, NY: Ronald Press.1922

DE MORAES, C. C. et al. Does resilience influence food waste causes? A systematic literature review. Gestao e Producao, v. 26, n. 3, p. 1-17, 2019. 
ELLWOOD, L. et al. Effectiveness of flavonoid rich fruits for hypertension in adults: A systematic review protocol. JBI Database of Systematic Reviews and Implementation Reports, v. 16, n. 11, p. 2103-2108, 2018.

FALLAH, A. A.; SARMAST, E.; JAFARI, T. Effect of dietary anthocyanins on biomarkers of glycemic control and glucose metabolism: A systematic review and meta-analysis of randomized clinical trials. Food Research International, v. 137, n. June, p. 109379, 2020. Disponível em: <https://doi.org/10.1016/j.foodres.2020.109379>.

FERENHOF, H. A.; FERNANDES, R. F. Demystifying the Literature Review As a Basis Forscientific Writing: Ssf Method. Revista ACB: Biblioteconomia em Santa Catarina, v. 21, n. 3, p. 550-563, 2016.

GAFFEY, A. et al. The effects of curcuminoids on musculoskeletal pain: A systematic review. JBI Database of Systematic Reviews and Implementation Reports, v. 15, n. 2, p. 486-516, 2017.

GRAZIOSI, MARIA ELISABETE SALVADOR; LIEBANO, RICHARD ELOIN; NAHAS, F. X. Elaboração da pergunta norteadora de pesquisa. In: UNASUS (Ed.). Módulo científico Especialização e Saúde da Família - Modalidade a distância. São Paulo: Unifesp Universidade Federal de São Paulo, 2010.

GREENHALGH, T. Papers that summarise other papers (systematic reviews and metaanalyses). British Medical Journal, v. 315, n. 7109, p. 672-675, 1997.

INGRAM, LAURA; HUSSEY, JAMES; TIGANI, MICHELLE; HEMMELGARN, M. Writing A Literature Review and Using a Synthesis Matrix MyWriting \& Speaking - Tutorial services. [s.l: s.n.]. Disponível em: <https://case.fiu.edu/writingcenter/ onlineresources/_assets/synthesis-matrix-2.pdf>.

JESSON, J.K., MATHESON, L. LACEY, F.M. "Doing Your Literature Review: Traditional and Systematic Techniques”. Evaluation \& Research in Education. v. 24, n.3, 2011.

MENDES, K. D. S.; SILVEIRA, R. C. de C. P.; GALVÃO, C. M. Revisão integrativa: método de pesquisa para a incorporação de evidências na saúde e na enfermagem. Texto \& Contexto - Enfermagem, v. 17, n. 4, p. 758-764, 2008. 
MOHER, DAVID; LIBERATI, ALESSANDRO; TETZLAFF, JENNIFER; ALTMAN, D. G. . T. P. G. Preferred Reporting Items for Systematic Revieews and Meta-Analyses: The PRISMA Statement. PLoS Medicine, v. 6, n. 7, p. 1-6, 2009.

PACHECO, J. C.; ARAÚJO, J. I. da S.; SANTANA, A. Análises de redes sociais em projetos: um mapeamento sistemático com foco na estrutura de times em projetos de software. Revista Brasileira de Administração Científica v.4, n.2. Ago, 2013

PETERS, M.D.J. GODFREY, C. MCINERNEY, P. SOARES, B. C. KHALIL, H., PARKER, D. Chapter 11: Scoping Reviews. In: Aromataris E, Munn Z (Editors). Joanna Briggs Institute Reviewer's Manual. The Joanna Briggs Institute, 2017. Available from https://reviewersmanual.joannabriggs.org/

PILKINGTON, A.; MEREDITH, J. The evolution of the intellectual structure of operations management-1980-2006: A citation/co-citation analysis. Journal of Operations Management, v. 27, n. 3, p. 185-202, 2009.

REIS, A. V. dos. Como fazer uma Revisão Sistemática \& Análise Bibliométrica (versão resumida) Nao Tem, p. 9, [s.d.]Disponível em: <https://www.google.com.br>.

ROSALEM, A. Como criar o gráfico de Gantt no Excel (publicado em 03/03/2013). Disponível em: http://msoexcel.blogspot.com/2013/03/como-criar-o-grafico-de-gantt-noexcel.html. Acesso em 25/01/2021.

SLACK, N.; BRANDON-JONES, A.; JOHNSTON, R. Administração da produção. 8.ed. Editora Atlas, 2018.

TOFANELLI, M. B. D.; WORTMAN, S. E. Benchmarking the agronomic performance of biodegradable mulches against polyethylene mulch film: A meta-analysis. Agronomy, v. 10, n. 10 October, 2020.

Autor para correspondência: Sila Mary Rodrigues Ferreira e-mail: sila.ufpr@gmail.com Departamento de Nutrição da Universidade Federal do Paraná (UFPR) Recebido: 23/02/2021 Aceite: 20/05/2021 